\title{
NUMERICAL COMPUTATION OF DOUBLE SURFACE INTEGRALS OVER TRIANGULAR CELLS FOR VORTEX SHEET INTENSITY RECONSTRUCTION ON BODY SURFACE IN 3D VORTEX METHODS
}

\author{
ILIA MARCHEVSKY ${ }^{1,2} \&$ GEORGY SHCHEGLOV ${ }^{1,2}$ \\ ${ }^{1}$ Bauman Moscow State Technical University, Russia \\ ${ }^{2}$ Ivannikov Institute for System Programming of the RAS, Russia
}

\begin{abstract}
In this paper, a new approach is developed for the computation of vortex sheet intensity in vortex methods for 3D flow simulation. The problem is reduced to a boundary integral equation of the second kind on the body surface with respect to an unknown vector variable. The proposed technique makes it possible to improve the accuracy significantly in comparison to the technique traditionally implemented in vortex methods. A Galerkin-type approach is used with piecewise-constant basis functions. The coefficients of the resulting algebraic system are expressed through double surface integrals, calculated over the mesh cells. A semi-analytic technique is developed for the integrals calculation; integration over one cell is carried out analytically, while at the integration over the other cell (having common edge or vertex with the first one), the integrand is singular. Analytic expressions are obtained for singular parts of integrands and for the results of their integration. The regular parts of the integrands are integrated numerically. The developed approach provides less than $0.1 \%$ error. The regularization technique is developed for a divergence-free vortex sheet reconstruction on the body surface. The developed approach works well on coarse and non-uniform surface meshes for complex-shaped bodies, which is important for engineering applications.
\end{abstract}

Keywords: vortex methods, double layer potential, vortex sheet, boundary integral equation, singularity exclusion, numerical integration.

\section{INTRODUCTION}

The problem of 3D incompressible flow simulation around rigid and immovable body is considered. The governing equations for the outer flow around the body are the Navier-Stokes equations with the no-slip boundary conditions on the body surface $K$ and perturbation decay conditions on infinity [1].

It is well-known that in order to take into account the presence of the body in the flow, it is possible to replace it with the vortex sheet of unknown intensity $\gamma(\mathbf{r})$, placed on the body surface, $\mathbf{r} \in K$, which generates the velocity field $\mathbf{V}_{\gamma}(\mathbf{r})$. Than the summary velocity field is the superposition of the incident flow velocity $\mathbf{V}_{\infty}$, velocity field, generated by vorticity in the flow domain $\mathbf{V}_{\Omega}(\mathbf{r})$, and the introduced field $\mathbf{V}_{\gamma}(\mathbf{r})$ :

$$
\mathbf{V}(\mathbf{r})=\mathbf{V}_{\infty}+\mathbf{V}_{\Omega}(\mathbf{r})+\mathbf{V}_{\gamma}(\mathbf{r}), \quad \mathbf{r} \in S,
$$

where $S$ is fluid domain, velocities $\mathbf{V}_{\Omega}(\mathbf{r})$ and $\mathbf{V}_{\gamma}(\mathbf{r})$ can be reconstructed through vorticity distribution according to the Biot-Savart law:

$$
\mathbf{V}_{\Omega}=\int_{S} \frac{\Omega(\xi) \times(\mathbf{r}-\xi)}{4 \pi|\mathbf{r}-\xi|^{3}} d S_{\xi}, \quad \mathbf{V}_{\gamma}=\oint_{K} \frac{\gamma(\xi) \times(\mathbf{r}-\xi)}{4 \pi|\mathbf{r}-\xi|^{3}} d K_{\xi} .
$$


From a mathematical point of view, the potential of the velocity field $\mathbf{V}_{\gamma}$ can be expressed through unknown double layer potential density $g(\mathbf{r})$ [2]:

$$
\mathbf{V}_{\gamma}(\mathbf{r})=\nabla \Phi(\mathbf{r})=\oint_{K} g(\xi) \frac{\partial}{\partial \mathbf{n}(\mathbf{r})} \frac{\partial}{\partial \mathbf{n}(\xi)} \frac{1}{4 \pi|\mathbf{r}-\xi|} d S_{\xi} .
$$

Note that the velocity field, which corresponds to this potential, can also be written down in the following form [2]:

$$
\mathbf{V}_{\gamma}(\mathbf{r})=\nabla \Phi(\mathbf{r})=\oint_{K} \frac{\gamma(\xi) \times(\mathbf{r}-\xi)}{4 \pi|\mathbf{r}-\xi|^{3}} d S_{\xi}
$$

where vector $\gamma(\mathbf{r})=-\operatorname{Grad} g(\mathbf{r}) \times \mathbf{n}(\mathbf{r})$; "Grad" means surface gradient operator. One can notice, that the expression (3) coincides with the Biot-Savart law for incompressible flows. So the potential $g(\mathbf{r})$ is closely connected with vortex sheet intensity $\gamma(\mathbf{r})$.

The velocity field $\mathbf{V}(\mathbf{r})$ given by (1), has discontinuity at the body surface; its limit value in the considered case of rigid immovable body is the following:

$$
\mathbf{V}_{-}(\mathbf{r})=\mathbf{V}(\mathbf{r})-\frac{\operatorname{Grad} g(\mathbf{r})}{2}=\mathbf{V}(\mathbf{r})-\frac{\gamma(\mathbf{r}) \times \mathbf{n}(\mathbf{r})}{2}, \quad \mathbf{r} \in K
$$

Taking into account the no-slip boundary condition in the form $\mathbf{V}_{-}=\mathbf{0}$ at the body surface, we obtain from (2) and (3) two forms of the integral equation:

$$
\oint_{K} g(\xi) \frac{\partial}{\partial \mathbf{n}(\mathbf{r})} \frac{\partial}{\partial \mathbf{n}(\xi)} \frac{1}{4 \pi|\mathbf{r}-\xi|} d S_{\xi}-\frac{\operatorname{Grad} g(\mathbf{r})}{2}=-\left(\mathbf{V}_{\infty}+\mathbf{V}_{\Omega}(\mathbf{r})\right), \quad \mathbf{r} \in K,
$$

or

$$
\oint_{K} \frac{\gamma(\xi) \times(\mathbf{r}-\xi)}{4 \pi|\mathbf{r}-\xi|^{3}} d S_{\xi}-\frac{\gamma(\mathbf{r}) \times \mathbf{n}(\mathbf{r})}{2}=-\left(\mathbf{V}_{\infty}+\mathbf{V}_{\Omega}(\mathbf{r})\right), \quad \mathbf{r} \in K .
$$

It is proven in Kempka et al. [3], that in order to satisfy vector integral eqs (4) and (5), it is enough to satisfy the corresponding equations, being projected either onto surface normal unit vector or the tangential plane. According to the first approach, i.e., satisfying the boundary condition for normal velocity components, we obtain scalar boundary integral equation, while in the framework of projection onto the tangential plane the equation remains vectorial.

\section{DIRECT RECONSTRUCTION OF THE DOUBLE LAYER POTENTIAL DENSITY}

The most common approach, which is normally used in vortex methods, is the equation (4) projection onto normal unit vector, that leads to the hypersingular integral equation with respect to the double layer potential. Its approximate solution as a rule is considered to be piecewise-constant distribution of the double layer density on surface mesh cells. The efficient numerical algorithm for calculation of hypersingular integrals in sense of the Hadamard principal values is developed and proved by Lifanov et al. [2].

The idea of this algorithm is based on the fact, that the $i$-th polygonal panel with double layer potential density $g_{i}=$ const put exactly the same contribution $\mathbf{V}_{\gamma}^{(i)}$ to the velocity field given by (1) as a closed vortex filament with circulation $\Gamma_{i}=g_{i}$, placed on the panel 
circumfery. So it is possible to consider that the body surface is replaced by system of closed vortex lines, and the corresponding vorticity distribution is divergence-free, so it corresponds to the Helmholtz fundamental theorems [4]. A similar approach is used in Timofeev [5].

Numerical experiments show this approach works satisfactory for flows simulation around smooth bodies of rather simple shape, when the surface mesh is close to uniform. However, even in this case the directions of vortex line on the body surface is determined by the mesh topology, and can differ significantly from true vorticity surface distribution. This can lead to significant error in velocity field reconstruction in neighborhood of the body surface, that in turn leads to error in pressure distribution reconstruction. The mentioned problems can be partially overcome by representing of the surface vorticity distribution as a system of closed vortex filaments - vortex loops, which positions and circulations can be found according to [6]-[8] based on known double layer potential distribution.

Such approach works perfect, for example, in computer graphics applications [6], where it is enough to provide only qualitative results and high accuracy is not required. Its usage for flow simulation and hydrodynamic forces calculation is restricted, again, with rather simple body geometries and uniform meshes [7]. For essentially non-uniform meshes main problems are connected with the poor accuracy of the hypersingular boundary equation numerical solution and the large error, that can arise from re-interpolation procedure due to high cells area ratio.

\section{VORTEX SHEET INTENSITY RECONSTRUCTION}

The other way to the boundary condition satisfaction is developed in Kempka et al. [3] and it supposes the eqn (5) projection onto the tangential plane. Such approach leads to the vector boundary integral equation of the second kind,

$$
\mathbf{n}(\mathbf{r}) \times\left(\oint_{K} \frac{\gamma(\xi) \times(\mathbf{r}-\xi)}{4 \pi|\mathbf{r}-\xi|^{3}} d S_{\xi} \times \mathbf{n}(\mathbf{r})\right)-\frac{\gamma(\mathbf{r}) \times \mathbf{n}(\mathbf{r})}{2}=\mathbf{f}(\mathbf{r}), \quad \mathbf{r} \in K,
$$

where the right-hand side $\mathbf{f}(\mathbf{r})$ is known vector function, which in the case of immovable body surface depends on the vortex wake influence and the incident flow velocity:

$$
\mathbf{f}(\mathbf{r})=-\mathbf{n}(\mathbf{r}) \times\left(\left(\mathbf{V}_{\infty}+\mathbf{V}_{\Omega}(\mathbf{r})\right) \times \mathbf{n}(\mathbf{r})\right) .
$$

Note, that the kernel of the equation (6) is unbounded when $|\mathbf{r}-\xi| \rightarrow 0$.

In order to solve it numerically with high accuracy the following assumptions are introduced:

1. The body surface is discretized into $N$ triangular panels $K_{i}$ with areas $A_{i}$ and unit normal vectors $\mathbf{n}_{i}, i=1, \ldots, N$.

2. The unknown vortex sheet intensity on the $i$-th panel is assumed to be constant vector $\gamma_{i}, i=1, \ldots, N$, which lies in the plane of the $i$-th panel, i.e., $\gamma_{i} \cdot \mathbf{n}_{i}=0$.

3. The integral equation (6) is satisfied on average over the panels, or, the same, in Galerkin sense: its residual is orthogonal to the basis functions, where the $j$-th basis function is equal to 1 on the $j$-th panel and equal to 0 on all other panels.

According to these assumptions, the discrete analogue of the eqn (6) can be derived:

$$
\frac{1}{A_{i}} \sum_{j=1}^{N} \int_{K_{i}}\left(\int_{K_{j}}\left(\mathbf{n}_{i} \times \frac{\gamma_{j} \times(\mathbf{r}-\xi)}{4 \pi|\mathbf{r}-\xi|^{3}}\right) d S_{\xi} \times \mathbf{n}_{i}\right) d S_{r}-\frac{\boldsymbol{\gamma}_{i} \times \mathbf{n}_{i}}{2}=\frac{1}{A_{i}} \int_{K_{i}} \mathbf{f}(\mathbf{r}, t) d S_{r}, \quad i=1, \ldots, N .
$$


To write down the eqn (7) in the form of a linear algebraic system, we choose local orthonormal basis on every cell $\left(\tau_{i}^{(1)}, \tau_{i}^{(2)}, \mathbf{n}_{i}\right)$, where tangent vectors $\tau_{i}^{(1)}, \tau_{i}^{(2)}$ can be chosen arbitrary (in the plane of the cell) and $\tau_{i}^{(1)} \times \tau_{i}^{(2)}=\mathbf{n}_{i}$, so $\gamma_{i}=\gamma_{i}^{(1)} \tau_{i}^{(1)}+\gamma_{i}^{(2)} \tau_{i}^{(2)}$, and we can project (7) for every $i$-th panel onto directions $\tau_{i}^{(1)}$ and $\tau_{i}^{(2)}$ [9]-[11].

Note, that the obtained algebraic system has infinite set of solutions; in order to select the unique solution, the additional condition for the total vorticity (the integral from the vortex sheet intensity over the body surface) should be satisfied:

$$
\oint_{K} \gamma(\mathbf{r}, t) d S_{r}=\mathbf{0},
$$

which can be easily written down in the discretized form.

The resulting algebraic system is overdetermined, so it should be regularized similarly to [2] by introducing the regularization vector $\mathbf{R}=\left(R_{1}, R_{2}, R_{3}\right)^{T}$ :

$$
\left.\begin{array}{l}
\frac{1}{A_{i}} \boldsymbol{\tau}_{i}^{(1)} \cdot\left(\sum_{j=1}^{N} \gamma_{j}^{(1)} \boldsymbol{v}_{i j}^{(1)}+\sum_{j=1}^{N} \gamma_{j}^{(2)} \boldsymbol{v}_{i j}^{(2)}\right)-\frac{\gamma_{i}^{(2)}}{2}+\mathbf{R} \cdot \tau_{i}^{(2)}=\frac{b_{i}^{(1)}}{A_{i}}, \\
\frac{1}{A_{i}} \boldsymbol{\tau}_{i}^{(2)} \cdot\left(\sum_{j=1}^{N} \gamma_{j}^{(1)} \boldsymbol{v}_{i j}^{(1)}+\sum_{j=1}^{N} \gamma_{j}^{(2)} \boldsymbol{v}_{i j}^{(2)}\right)+\frac{\gamma_{i}^{(1)}}{2}+\mathbf{R} \cdot \tau_{i}^{(1)}=\frac{b_{i}^{(2)}}{A_{i}}, i=1, \ldots, N, \\
\sum_{j=1}^{N} A_{j}\left(\gamma_{j}^{(1)} \tau_{j}^{(1)}+\gamma_{j}^{(2)} \tau_{j}^{(2)}\right)=0,
\end{array}\right\}
$$

where the coefficients $v_{i j}^{(k)}$ and $b_{i}^{k}, i, j=1, \ldots, N, k=1,2$, are expressed as the following:

$$
\boldsymbol{v}_{i j}^{(k)}=\int_{K_{i}}\left(\int_{K_{j}} \frac{\tau_{j}^{(k)} \times(\mathbf{r}-\xi)}{4 \pi|\mathbf{r}-\xi|^{3}} d S_{\xi}\right) d S_{r}, \quad b_{i}^{(k)}=\int_{K_{i}} \tau_{i}^{(k)} \cdot \mathbf{f}(\mathbf{r}) d S_{r} .
$$

Similar approach, based on the projection of the boundary integral equation onto the tangential direction, has been investigated in Kuzmina et al. [12] for 2D modifications of vortex methods, and it is shown, that it allows for significant improvement of the accuracy of numerical solution. It should be noted, that in 2D case the resulting linear algebraic system has $(N+1) \times(N+1)$ size $(N$ is number of panels on the 2D airfoil surface line discretization; the regularization variable $R$ is scalar), but in the discussed $3 \mathrm{D}$ case matrix size is twice as number of panels: $(2 N+3) \times(2 N+3)$.

In contrast to $2 \mathrm{D}$ case, when the exact analytic expressions can be derived [13] for all the coefficients of the linear system, which is an analogue of the system (8), for 3D case it is impossible to calculate the integrals in expressions (9), but it is possible to develop efficient semi-analytical algorithm [9]-[11], which provides rather high accuracy.

\section{SEMI-ANALYTICAL TECHNIQUE FOR THE COEFFICIENTS CALCULATION}

The coefficients $\boldsymbol{v}_{i j}^{(k)}$ of the linear system (8) should be calculated for all the pairs of the panels on the surface mesh; however note, that $\boldsymbol{v}_{i i}^{(k)}=\mathbf{0}$ due to the flatness of the panels, while their self-influence is taken into account through the second (non-integral) term the eqn (7), which corresponds to the terms $\pm \gamma_{i}^{(k)} / 2$ in (8). In the case of $i \neq j$ let us denote: 


$$
\mathbf{v}_{i j}^{(k)}=\tau_{j}^{(k)} \times \int_{K_{i}}\left(\int_{K_{j}} \frac{\mathbf{r}-\xi}{4 \pi|\mathbf{r}-\xi|^{3}} d S_{\xi}\right) d S_{r}=\mathbf{I}_{i j} \times \tau_{j}^{(k)}, \quad k=1,2, \quad i, j=1, \ldots, N .
$$

The integral $\mathbf{I}_{i j}$ in (10) is calculated over the triangular panels $K_{i}$ and $K_{j}$; the $i$-th panel we call hereinafter "control panel", and the $k$-th one - "influence panel".

The problem, that is very similar to calculation of the $\mathbf{I}_{i j}$ coefficients, arises in the boundary element method (BEM, [14]). There are some known approaches to approximate calculation of such integrals, for example the Taylor - Duffy method, but the corresponding numerical procedures have high computational cost [15].

The inner integral in (10), calculated over the influence panel $K_{j}$,

$$
\mathbf{J}_{j}(\mathbf{r})=-\int_{K_{j}} \frac{\mathbf{r}-\xi}{4 \pi|\mathbf{r}-\xi|^{3}} d S_{\xi},
$$

can be calculated analytically using computer algebra system, such as Wolfram Mathematica, or some classic handbooks [16]. In order to write down the resulting formula for it, note that it is expressed through the vectors $\mathbf{s}_{k}=\mathbf{r}_{k}^{(j)}-\mathbf{r}, k=1,2,3$, where $\mathbf{r}$ is the position vector of the observation point, at which the integral (11) is calculated; $\mathbf{r}_{k}^{(j)}$ are position vectors of the vertices of the influence panel $K_{j}$. Introducing unit vectors (hereinafter we denote the unit vector, corresponding to arbitrary vector $\mathbf{r}$ as $\operatorname{ort}(\mathbf{r})=\mathbf{r} /|\mathbf{r}|)$,

$$
\mathbf{e}_{k}^{(j)}=\operatorname{ort}\left(\mathbf{s}_{k+1}-\mathbf{s}_{k}\right)=\operatorname{ort}\left(\mathbf{r}_{k+1}^{(j)}-\mathbf{r}_{k}^{(j)}\right), \quad \sigma_{k}=\operatorname{ort}\left(\mathbf{s}_{k}\right), \quad k=1,2,3,
$$

and having in mind that all indices are calculated in modulo 3, the expression for (11) now is:

$$
\mathbf{J}_{j}(\mathbf{r})=\frac{1}{4 \pi}\left(\Theta_{j} \mathbf{n}_{j}+\Psi_{j} \times \mathbf{n}_{j}\right), \quad j=1, \ldots, N,
$$

where $\Theta_{j}$ means the signed solid angle at which the panel $K_{j}$ is observed, equal to [17]:

$$
\Theta_{j}=2 \operatorname{atan}\left(\mathbf{s}_{1} \mathbf{s}_{2} \mathbf{s}_{3},\left|\mathbf{s}_{1}\right| \cdot\left|\mathbf{s}_{2}\right| \cdot\left|\mathbf{s}_{3}\right|+\left(\mathbf{s}_{1} \cdot \mathbf{s}_{2}\right)\left|\mathbf{s}_{3}\right|+\left(\mathbf{s}_{2} \cdot \mathbf{s}_{3}\right)\left|\mathbf{s}_{1}\right|+\left(\mathbf{s}_{3} \cdot \mathbf{s}_{1}\right)\left|\mathbf{s}_{2}\right|\right),
$$

where $\mathbf{s}_{1} \mathbf{s}_{2} \mathbf{s}_{3}$ means scalar triple product of the vectors. The function $\phi=\operatorname{atan}(y, x)$ hereinafter corresponds to usually used in programming languages atan $2(y, x)$ function, which means in turn the argument of a complex number $z=x+i y$, or, the same, the angle from the interval $\phi \in(-\pi, \pi]$, for which $\sin \phi=y / \sqrt{x^{2}+y^{2}}, \cos \phi=x / \sqrt{x^{2}+y^{2}}$.

The vector-function $\Psi_{j}$ has the form $\Psi_{j}=\sum_{k=1}^{3} \ln \psi_{k}^{(j)} \mathbf{e}_{k}$, where

$$
\psi_{k}^{(j)}=\frac{\left|\mathbf{s}_{k}\right|}{\left|\mathbf{s}_{k+1}\right|}\left(\frac{1+\mathbf{e}_{k}^{(j)} \cdot \sigma_{k}}{1+\mathbf{e}_{k}^{(j)} \cdot \sigma_{k+1}}\right)
$$

except the case $\mathbf{e}_{k}^{(j)} \cdot \boldsymbol{\sigma}_{k}=\mathbf{e}_{k}^{(j)} \cdot \boldsymbol{\sigma}_{k+1}=-1$, for which the limit value is $\psi_{k}^{(j)}=\frac{\left|\mathbf{s}_{k+1}\right|}{\left|\mathbf{s}_{k}\right|}$. 
4.1 The outer integral calculation in the general case

The outer integral in (10) over the control mesh cell,

$$
\mathbf{I}_{i j}=\int_{K_{i}} \mathbf{J}_{j}(\mathbf{r}) d S_{r}
$$

cannot be analytically expressed through elementary functions in general case, so for the approximate computation of the vectors $\mathbf{I}_{i j}$ Gaussian quadrature formulae can be used:

$$
\mathbf{I}_{i j}=\int_{K_{i}} \mathbf{J}_{j}(\mathbf{r}) d S_{r} \approx A_{i} \sum_{p=1}^{N_{G P}} \omega_{p} \mathbf{J}_{j}\left(\eta_{p}\right),
$$

where $N_{G P}$ is number of Gaussian points; $\omega_{p}$ are weights; $\eta_{p}$ are positions of the Gaussian points for triangular mesh cell [18].

The described approach for integrals $\mathbf{I}_{i j}$ computation is applicable, however, only for those pairs of the panels, which are non-neighboring, i.e., if they do not have common edges and vertices, because in the case of neighboring panels the integrand in (13) becomes unbounded in proximity to the common edge/vertex, and the outer integral itself become improper (but convergent). Its calculation with high precision straightforwardly by using quadrature formulae is difficult, so it is suggested to exclude the singularity from the integrand. We write down the integrand $\mathbf{J}_{j}(\mathbf{r})$ as a sum of two terms

$$
\mathbf{J}_{j}(\mathbf{r})=\mathbf{J}_{j}^{\mathrm{reg}}(\mathbf{r})+\mathbf{J}_{j}^{\text {sing }}(\mathbf{r})
$$

where the regular part

$$
\mathbf{J}_{j}^{\mathrm{reg}}(\mathbf{r})=\frac{1}{4 \pi}\left(\left(\Theta_{j}(\mathbf{r})-\Theta_{j}^{\mathrm{sing}}(\mathbf{r})\right) \mathbf{n}_{j}+\left(\Psi_{j}(\mathbf{r})-\Psi_{j}^{\mathrm{sing}}(\mathbf{r})\right) \times \mathbf{n}_{j}\right)
$$

is a smooth function, which can be easily integrated numerically by using above mentioned Gaussian quadratures. For the singular part it is possible to integrate analytically the following terms:

$$
\int_{K_{i}} \mathbf{J}_{j}^{\text {sing }}(\mathbf{r}) d S_{r}=\frac{1}{4 \pi}\left(\left(\int_{K_{i}} \Theta_{j}^{\text {sing }}(\mathbf{r}) d S_{r}\right) \mathbf{n}_{j}+\left(\int_{K_{i}} \Psi_{j}^{\text {sing }}(\mathbf{r}) d S_{r}\right) \times \mathbf{n}_{j}\right) .
$$

4.2 The outer integral calculation in the case of common edge

If the panels $K_{i}$ and $K_{j}$ have a common edge with unit direction vector $\mathbf{e}_{3}$ (Fig. 1), the singular terms have the form (the upper index $(j)$ for the unit vectors $\mathbf{e}_{k}^{(j)}$ is omitted):

$$
\begin{gathered}
\Theta_{j}^{\text {sing }}(\mathbf{r})=2 \sum_{k=1}^{2} \operatorname{atan}\left(\mathbf{a}_{k} \mathbf{e}_{k} \mathbf{e}_{3},\left(\mathbf{e}_{3}-\mathbf{e}_{k}\right) \cdot\left(\mathbf{a}_{k}-\mathbf{e}_{k}\right)\right), \\
\Psi_{j}^{\text {sing }}(\mathbf{r})=\mathbf{e}_{3} \ln \left(\frac{m_{1}}{m_{2}} \frac{\mathbf{e}_{3} \cdot\left(\mathbf{e}_{3}+\mathbf{a}_{1}\right)}{\mathbf{e}_{3} \cdot\left(\mathbf{e}_{3}-\mathbf{a}_{2}\right)}\right)+\sum_{k=1}^{2} \mathbf{e}_{k} \ln \left(\frac{m_{k}}{m_{3}} \mathbf{e}_{k} \cdot\left(\mathbf{e}_{k}-\mathbf{a}_{k}\right)\right),
\end{gathered}
$$

where $m_{1}=\left|\mathbf{r}-\mathbf{r}_{1}^{(j)}\right|, m_{2}=\left|\mathbf{r}-\mathbf{r}_{3}^{(j)}\right|, m_{3}=\left|\mathbf{r}_{1}^{(j)}-\mathbf{r}_{3}^{(j)}\right|, \mathbf{a}_{1}=\operatorname{ort}\left(\mathbf{r}-\mathbf{r}_{1}^{(j)}\right), \mathbf{a}_{2}=-\operatorname{ort}\left(\mathbf{r}-\mathbf{r}_{3}^{(j)}\right)$. 


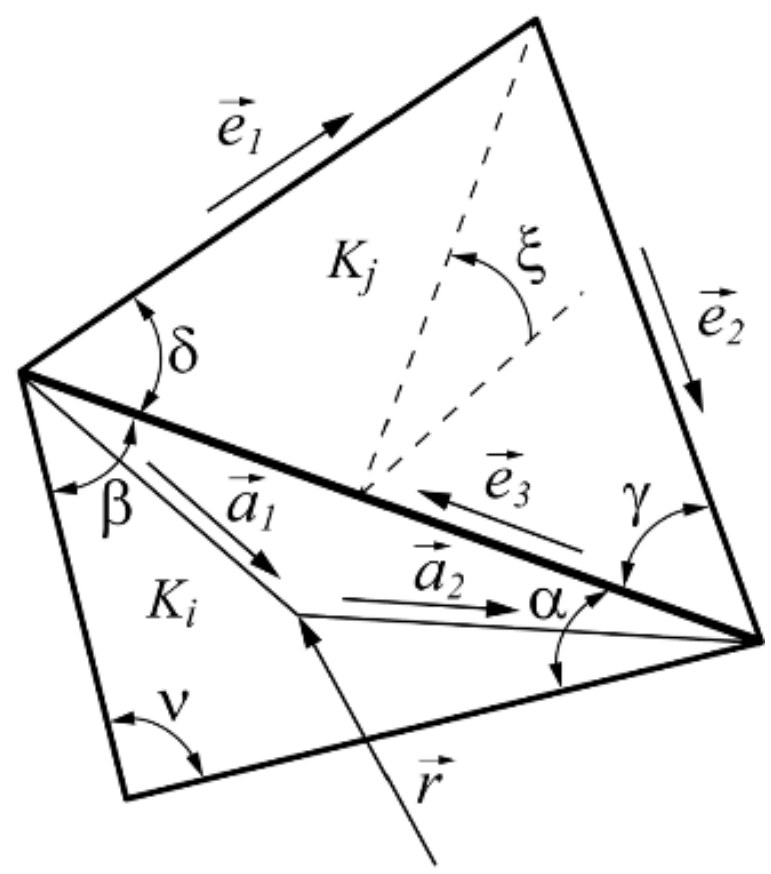

Figure 1: Mesh cells with common edge.

The expression for $\Theta_{j}^{\text {sing }}$, as well as for scalar multipliers in $\Psi_{j}^{\text {sing }}$, can be integrated analytically over the control panel $K_{i}$ :

$$
\begin{gathered}
\int_{K_{i}} \Theta_{j}^{\text {sing }}(\mathbf{r}) d S_{r}=-2 A_{i}\left(q_{0}(\xi, \alpha, \beta, \mu, \gamma, \lambda)+q_{0}(\xi, \beta, \alpha, \sigma, \delta, \theta)\right) \\
\int_{K_{i}} \Psi_{j}^{\text {sing }}(\mathbf{r}) d S_{r}=A_{i}\left(q_{12}(\xi, \alpha, \beta, \mu, \gamma, \lambda) \mathbf{e}_{1}+q_{12}(\xi, \beta, \alpha, \sigma, \delta, \theta) \mathbf{e}_{2}+q_{3}(\alpha, \beta) \mathbf{e}_{3}\right) .
\end{gathered}
$$

The expressions for the auxiliary functions $q_{0}, q_{12}$ and $q_{3}$ have the following form:

$$
\begin{gathered}
q_{0}(\xi, \alpha, \beta, \mu, \gamma, \lambda)=\phi^{*}+\frac{\sin \gamma \sin v}{\sin ^{2} \mu \sin \alpha}\left((\cos \beta \sin \gamma-\sin \beta \cos \gamma \cos \xi) \phi^{* *}+\right. \\
\left.+\sin \xi \sin \beta\left(\cos ^{2} \frac{\mu}{2} \ln \frac{\cos \beta / 2}{\sin v / 2}+\sin ^{2} \frac{\mu}{2} \ln \frac{\sin \beta / 2}{\cos v / 2}+\ln \frac{\cos \lambda / 2}{\sin \gamma / 2}\right)\right) \\
q_{12}(\xi, \alpha, \beta, \mu, \gamma, \lambda)=-\frac{3}{2}+\frac{1}{\sin \alpha \sin ^{2} \mu}(\sin \beta(\cos v+\cos \mu \cos \lambda) \ln (1+\cos \lambda)+ \\
+\sin v(\cos \beta+\cos \gamma \cos \mu) \ln (1-\cos \gamma)+\sin \beta(1-\cos \mu)(\cos v-\cos \lambda) \ln \frac{\sin \beta}{\sin v}-
\end{gathered}
$$




$$
\begin{array}{r}
\left.-\sin v \sin \beta\left(-2 \phi^{* *} \sin \xi \sin \gamma+(\sin \gamma \cos \beta \cos \xi-\sin \beta \cos \gamma) \ln \frac{1-\cos v}{1+\cos \beta}\right)\right) \\
q_{3}(\alpha, \beta)=\frac{\sin v}{\sin \beta} \ln \left(\tan \frac{\alpha}{2} \tan \frac{v}{2}\right)+\frac{\sin v}{\sin \alpha} \ln \left(\tan \frac{\beta}{2} \tan \frac{v}{2}\right)+\ln \left(\tan \frac{\alpha}{2} \tan \frac{\beta}{2}\right) .
\end{array}
$$

Here $\alpha$ and $\beta$ are angles of the control triangular cell $K_{i}$, which adjoin the common edge; $v=\pi-\alpha-\beta ; \gamma$ and $\delta$ are angles of the influence triangular cell $K_{j}$, which adjoin the common edge; $\xi$ is the angle between the planes of the cells $K_{i}$ and $K_{j}$ (Fig. 1); the other angles are given by the expressions:

$\sigma=\pi-\arccos (\cos \alpha \cos \delta+\cos \xi \sin \alpha \sin \delta), \quad \mu=\pi-\arccos (\cos \beta \cos \gamma+\cos \xi \sin \beta \sin \gamma)$, $\lambda=\pi-\arccos (\cos \alpha \cos \gamma-\cos \xi \sin \alpha \sin \gamma), \quad \theta=\pi-\arccos (\cos \beta \cos \delta-\cos \xi \sin \beta \sin \delta)$, $\phi^{*}=\operatorname{atan}(\sin \xi \sin \alpha \sin \gamma, 1-\cos \alpha+\cos \gamma+\cos \lambda), \quad \phi^{* *}=\operatorname{atan}(\sin \xi \sin \alpha \sin \gamma, 1+\cos \alpha-\cos \gamma+\cos \lambda)$.

The given formulae are valid for arbitrary mutual configuration of the panels $K_{i}$ and $K_{j}$, except the particular case $\xi=\beta-\gamma=0$, for which they lead to uncertainty of $0 / 0$ type. However, carrying out the passage to the limit, it is possible to obtain for this case rather simple expressions for $q_{0}$ and $q_{12}$ functions:

$$
\begin{gathered}
q_{0}(\xi, \alpha, \beta, \mu, \gamma, \lambda)=\phi^{*}, \\
q_{12}(\xi, \alpha, \beta, \mu, \gamma, \lambda)=-\frac{3}{2}+\frac{1}{2 \sin \alpha}(\cos v \sin \beta \ln (1+\cos v)+\sin v \cos \beta \ln (1-\cos \beta)) .
\end{gathered}
$$

4.3 The outer integral calculation in the case of common vertex

The scheme, which illustrates the configuration of the panels having common vertex with introduced designations is shown in Fig. 2.

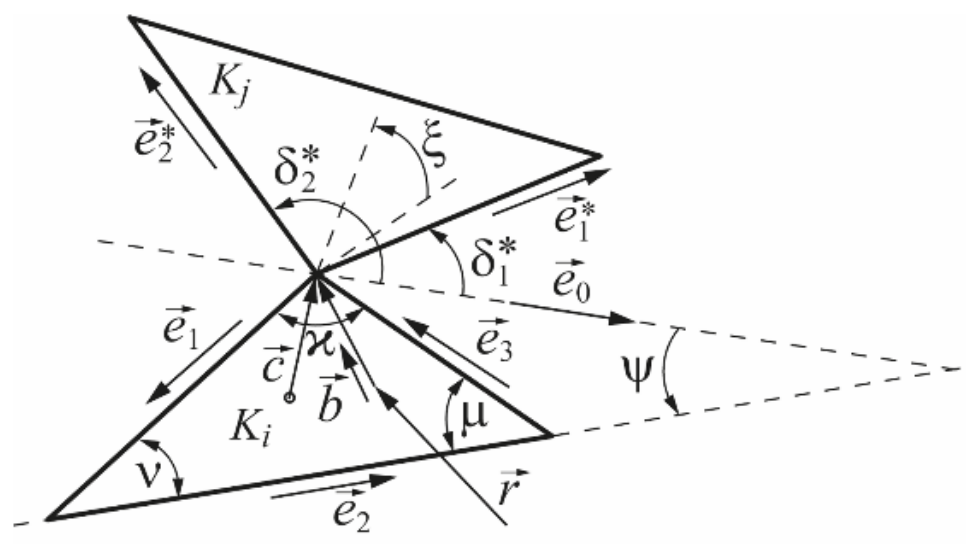

Figure 2: Mesh cells with common edge. 
The triangular panels $K_{i}$ and $K_{j}$ lie in planes, which intersection line has direction unit vector $\mathbf{e}_{0}$, collinear to the vector $\mathbf{n}_{i} \times \mathbf{n}_{j}$. The direction of the vector $\mathbf{e}_{0}$ is chosen in such a way, that scalar triple product $\mathbf{e}_{0} \mathbf{c n}_{i}>0$, where vector $\mathbf{c}$ connects the center of the panel $K_{i}$ with the common vertex. If the panels $K_{i}$ and $K_{j}$ are complanar, i.e., $\mathbf{n}_{i} \times \mathbf{n}_{j}=\mathbf{0}$, the direction of the unit vector $\mathbf{e}_{0}$ can be chosen arbitrary; to be more specific, we choose it as $\mathbf{e}_{0}=-\mathbf{e}_{1}$ if $\mathbf{e}_{2} \cdot \mathbf{e}_{3} \leq 0$ and $\mathbf{e}_{0}=\mathbf{e}_{3}$ if $\mathbf{e}_{2} \cdot \mathbf{e}_{3}>0$, where $\mathbf{e}_{k}$ are unit vectors, which are now directed along the edges of the control panel $K_{i}$ under the assumption, that the common vertex has index $k=1$ (i.e., its position vector is $\mathbf{r}_{1}^{(i)}$ ):

$$
\mathbf{e}_{k}=\operatorname{ort}\left(\mathbf{r}_{k+1}^{(i)}-\mathbf{r}_{k}^{(i)}\right), k=1,2,3 .
$$

The orientation of the influence panel $K_{j}$ is defined by the unit vectors

$$
\mathbf{e}_{1}^{*}=\operatorname{ort}\left(\mathbf{r}_{2}^{(j)}-\mathbf{r}_{1}^{(j)}\right), \mathbf{e}_{2}^{*}=\operatorname{ort}\left(\mathbf{r}_{3}^{(j)}-\mathbf{r}_{1}^{(j)}\right),
$$

and the angles:

$$
\delta_{k}^{*}=\angle\left(\mathbf{e}_{0}, \mathbf{e}_{k}^{*}\right)=\operatorname{atan}\left(\mathbf{e}_{0} \mathbf{e}_{k}^{*} \mathbf{n}_{j}, \mathbf{e}_{0} \cdot \mathbf{e}_{k}^{*}\right), k=1,2 .
$$

In particular cases when $\delta_{1}^{*}=\pi$ or $\delta_{2}^{*}=\pi$ the direction of the vector $\mathbf{e}_{0}$ should be reversed, and the angles $\delta_{k}^{*}$ should be recalculated for its new orientation. The same operations should be performed in the case, when $\delta_{1}^{*} \delta_{2}^{*}<0$ and $\left|\delta_{1}^{*}-\delta_{2}^{*}\right|>\pi$ simultaneously.

Since the vector $\mathbf{e}_{0}$ is defined, the signed angle between the planes $K_{i}$ and $K_{j}$ is:

$$
\xi=\operatorname{atan}\left(\mathbf{n}_{i} \mathbf{n}_{j} \mathbf{e}_{0}, \mathbf{n}_{i} \cdot \mathbf{n}_{j}\right),
$$

its sign defines the mutual orientation of the triangular cells.

Considering the singular part $\mathbf{J}_{j}^{\text {sing }}(\mathbf{r})$ of

$$
\mathbf{J}_{j}(\mathbf{r})=\frac{1}{4 \pi}\left(\Theta_{j} \mathbf{n}_{j}+\Psi_{j} \times \mathbf{n}_{j}\right),
$$

we write down the singular parts of its terms. For the first term

$$
\Theta_{j}^{\text {sing }}(\mathbf{r})=-2\left(\Theta_{2}^{*}-\Theta_{1}^{*}+\tilde{\Theta}\right)
$$

where $\Theta_{1}^{*}$ and $\Theta_{2}^{*}$ are expressed through vectors $\mathbf{u}_{k}^{*}=\mathbf{e}_{0}+\mathbf{e}_{k}^{*}, \mathbf{v}_{k}^{*}=\left(\mathbf{e}_{0}+\mathbf{e}_{k}^{*}\right) \times \mathbf{e}_{0}$ and the unit vector $\mathbf{b}=\operatorname{ort}\left(\mathbf{r}_{1}^{(i)}-\mathbf{r}\right)$ :

$$
\Theta_{k}^{*}=\operatorname{atan}\left(\mathbf{p}_{k} \cdot \mathbf{v}_{k}^{*}, \mathbf{p}_{k} \cdot \mathbf{u}_{k}^{*}\right)
$$

where

$$
\mathbf{p}_{k}=\left(\left(\mathbf{b}+\mathbf{e}_{0}\right) \cdot \mathbf{u}_{k}^{*}\right) \mathbf{u}_{k}^{*}+\left(\left(\mathbf{b}+\mathbf{e}_{0}\right) \cdot \mathbf{v}_{k}^{*}\right) \mathbf{v}_{k}^{*}, k=1,2 .
$$


For the particular case at $\left|\mathbf{p}_{k}\right|=0$ the limit value is $\Theta_{k}^{*}=\operatorname{atan}\left(\mathbf{b} \cdot \mathbf{n}_{j}, \mathbf{b n}_{j} \mathbf{e}_{0}\right)$. The term $\tilde{\Theta}=0$ at $\left|\mathbf{p}_{k}\right| \neq 0$ and $\tilde{\Theta}=\pi \operatorname{sign} \xi$ at $\left|\mathbf{p}_{k}\right|=0$.

For the singular part of the second term in $\mathbf{J}_{j}(\mathbf{r})$ we obtain

$$
\Psi_{j}^{\text {sing }}(\mathbf{r})=-\left(\Psi_{2}^{*}-\Psi_{1}^{*}\right)
$$

where

$$
\Psi_{k}^{*}=\mathbf{e}_{k}^{*} \ln \left(\frac{m_{b}}{\sqrt{A_{j}}} \mathbf{e}_{k}^{*} \cdot\left(\mathbf{b}+\mathbf{e}_{k}^{*}\right)\right), \quad m_{b}=\left|\mathbf{r}_{1}^{(i)}-\mathbf{r}\right| .
$$

As for the panels with common edge, the singular parts $\Theta_{j}^{\text {sing }}(\mathbf{r})$ and $\Psi_{j}^{\text {sing }}(\mathbf{r})$ can be integrated analytically over the control mesh cell $K_{i}$ :

$$
\begin{gathered}
\int_{K_{i}} \Theta_{j}^{\text {sing }}(\mathbf{r}) d S_{r}=-2 A_{i}\left(q_{4}\left(\delta_{2}^{*}\right)-q_{4}\left(\delta_{1}^{*}\right)+\tilde{q} q_{4}(0)\right), \\
\int_{K_{i}} \Psi_{j}^{\text {sing }}(\mathbf{r}) d S_{r}=-A_{i}\left(q_{5}\left(\delta_{2}^{*}\right) \mathbf{e}_{2}^{*}-q_{5}\left(\delta_{1}^{*}\right) \mathbf{e}_{1}^{*}\right),
\end{gathered}
$$

where the coefficient $\tilde{q}=0$ at $\delta_{1}^{*} \delta_{2}^{*} \geq 0$ and $\tilde{q}=-2 \operatorname{sign} \xi$ at $\delta_{1}^{*} \delta_{2}^{*}<0$, and the auxiliary functions $q_{4}$ and $q_{5}$ are the following:

$$
q_{4}(\delta)=\frac{q_{41}(\delta)-q_{42}(\delta)}{\sin \psi \sin \kappa}, \quad q_{5}(\delta)=q_{51}(\delta)-q_{52}(\delta) .
$$

The expressions for their terms are rather complicated, but they are expressed through elementary functions:

$$
\begin{gathered}
q_{41}(\delta)=\sin \mu \sin (v+\psi) \operatorname{atan}\left(\sin \xi \sin \frac{\delta}{2}, \cos \xi \sin \frac{\delta}{2}+\cos \frac{\delta}{2} \cot \frac{v+\psi}{2}\right)- \\
-\sin v \sin (\mu-\psi) \operatorname{atan}\left(\sin \xi \sin \frac{\delta}{2}, \cos \xi \sin \frac{\delta}{2}+\cos \frac{\delta}{2} \tan \frac{\mu-\psi}{2}\right), \\
q_{42}(\delta)=\frac{\sin \mu \sin v \sin \delta}{D}\left(\omega \cos \eta+\frac{\sin \psi \sin \xi}{2}\left(\ln \left(\frac{1+\cos \lambda}{1+\sin \theta} \frac{\sin \theta}{\sin \mu}\right)-\cos \sigma \ln \left(\tan \frac{v}{2} \tan \frac{\mu}{2}\right)\right),\right. \\
q_{51}(\delta)=-\frac{3-\ln 2}{2}+\frac{\sin \mu \sin (v+\psi) \ln (1+\cos \theta)-\sin v \sin (\mu-\psi) \ln (1+\cos \lambda)}{\sin \kappa \sin \psi}+ \\
+\frac{1}{2} \ln \left(\frac{\sin v \sin \mu}{\sin \kappa}\right)-\frac{\cos v \sin \mu}{\sin \kappa} \ln (\sin v)-\frac{\cos \mu \sin v}{\sin \kappa} \ln (\sin \mu), \\
q_{52}(\delta)=\frac{\sin v \sin \mu}{D \sin \kappa}\left(\frac{\sin \delta \cos \eta}{\sin \psi} \ln \frac{1+\cos \theta}{1+\cos \lambda}-\cos \chi \ln \left(\tan \frac{v}{2} \tan \frac{\mu}{2}\right)+\right. \\
\left.+2 \omega \sin \delta \sin \xi+\frac{1}{2} G \ln \frac{\sin v}{\sin \mu}\right) .
\end{gathered}
$$

Here the following designations are introduced: 


$$
\begin{array}{r}
D=\sin ^{2}(\delta-\psi)+4 \sin \delta \sin \psi\left(\sin \delta \sin \psi \cos ^{2} \frac{\xi}{2}+\cos \delta \cos \psi\right) \sin ^{2} \frac{\xi}{2}, \\
G=\sin 2(\delta-\psi)-4 \sin \delta\left(\sin \delta \sin 2 \psi \cos ^{2} \frac{\xi}{2}+\cos \delta \cos 2 \psi\right) \sin ^{2} \frac{\xi}{2} \\
\omega=\operatorname{atan}\left(\sin \xi \sin \delta \sin \frac{\kappa}{2}, \cos \xi \sin \delta \sin \left(\nu+\psi+\frac{\kappa}{2}\right)+\cos \frac{\kappa}{2}-\cos \delta \cos \left(\mu-\psi+\frac{\kappa}{2}\right)\right),
\end{array}
$$

value $\kappa$ means the angle of the triangular cell $K_{i}$ which adjoins to the common vertex of the panels $K_{i}$ and $K_{j} ; \mu$ and $v$ the other angles of the cell $K_{i} ; \xi$ as earlier is the signed angle between the planes of the triangles $K_{i}$ and $K_{j} ; \psi$ is the angle between the vector $\mathbf{e}_{0}$ and the side of the triangle $K_{i}$, which is opposite to the common vertex; $\delta_{1}^{*}$ and $\delta_{2}^{*}$ are the angles between the vector $\mathbf{e}_{0}$ and vectors $\mathbf{e}_{1}^{*}$ and $\mathbf{e}_{2}^{*}$, respectively (Fig. 2); for $\cos \sigma, \cos \lambda$, $\cos \theta, \cos \eta$ and $\cos \chi$ the following expressions are valid:

$$
\begin{array}{ll}
\cos \sigma=\sin \delta \sin \psi \cos \xi+\cos \delta \cos \psi, & \cos \theta=\sin \delta \sin (v+\psi) \cos \xi+\cos \delta \cos (v+\psi), \\
\cos \chi=\sin \delta \cos \psi \cos \xi-\cos \delta \sin \psi, & \cos \lambda=\sin \delta \sin (\mu-\psi) \cos \xi-\cos \delta \cos (\mu-\psi), \\
\cos \eta=\cos \delta \sin \psi \cos \xi-\sin \delta \cos \psi . &
\end{array}
$$

These formulae for the integrals from the singular part $\Psi_{j}^{\text {sing }}(\mathbf{r})$ remain correct for arbitrary mutual configuration of the triangular cells with common vertex except the case of $\xi=\sin (\delta-\psi)=0$. For this particular case:

$$
q_{42}(\delta)=0, \quad q_{52}(\delta)=\frac{1}{2}\left(\sin \psi\left(\frac{1+\cos v}{\sin v}-\frac{\sin \mu}{1+\cos \mu}\right)-\cos \psi \ln \frac{1-\cos v}{1+\cos \mu}\right) .
$$

\section{DOUBLE LAYER POTENTIAL RECONSTRUCTION}

After solution of the linear system (8) with respect to cell values of the vortex sheet intensity, it is important to provide divergence-free reconstruction of the solution. That means, that the numerical solution of (8) in general case doesn't satisfy the following condition, which is analogue of the condition of zero total vorticity flux $\sum_{q=1}^{3}\left(\gamma_{i}-\gamma_{i}^{\text {neib, } q}\right) l_{q}$, where $\gamma_{i}^{\text {neib, } q}$ is the vortex sheet intensity on the panel, which is neighbor to the $i$-th cell against the $q$-th edge; $l_{q}$ is the length of the $q$-th edge.

In order to satisfy this condition and provide divergence-free numerical solution, it is suggested firstly to reconstruct the double layer density distribution on the body surface, and secondly calculate its surface gradient. Moreover, if the double layer density distribution is known, it is easy to represent vorticity distribution on the body surface as a set vortex loops, that have been mentioned in Section 2.

The solution of the linear system (8) corresponds to piecewise-constant vortex sheet intensity distribution over the body surface. From the other side, vortex sheet intensity is surface gradient of the double layer potential density, multiplied by normal unit vector. It means, that the most convenient way to double layer potential density reconstruction is its approximation by a continuous function, which is piecewise-linear with respect to all 
coordinates on every panel. To do it, we consider the nodal values of the potential $g_{j}$, $j=1, \ldots, M$ to be unknown; $M$ is number of vertices of the surface mesh. Then the potential density can be represented as FEM-type interpolation using the 1-st order shape functions. Let us note positions of all the vertices of the surface mesh as $\boldsymbol{\rho}_{j}, j=1, \ldots, M$, and consider that the vertices of the $i$-th triangular panel have indices $p_{i}^{k}, k=1,2,3$. So the shape functions, defined on the $i$-th panel, coincide with barycentric coordinates on the mesh cell:

$$
\phi_{i}^{(k)}(\rho)=\frac{\left|\left(\rho_{p_{i}^{l}}-\rho\right) \times\left(\rho_{p_{i}^{m}}-\rho\right)\right|}{\left|\left(\rho_{p_{i}^{l}}-\rho_{p_{i}^{k}}\right) \times\left(\rho_{p_{i}^{l}}-\rho_{p_{i}^{k}}\right)\right|}, \quad \rho \in K_{i},
$$

where $(k, l, m)=(1,2,3)$, or $(2,3,1)$ or $(3,1,2)$.

Then the double layer density on the $i$-th panel is linear function with respect to $\rho$ and has the following form:

$$
g(\rho)=\sum_{k=1}^{3} g_{p_{i}^{k}} \phi_{i}^{(k)}(\rho), \quad \rho \in K_{i} .
$$

The surface gradient of this distribution of the double layer on the every $i$-th panel, multiplied by unit normal vector $\mathbf{n}_{i}$ gives thus the constant vector, which physical sense is approximate value of divergence-free vortex sheet intensity on the corresponding panel:

$$
\gamma_{i}^{*}=-\sum_{k=1}^{3} g_{p_{i}^{k}}\left(\operatorname{Grad} \phi_{i}^{(k)} \times \mathbf{n}_{i}\right), \quad \rho \in K_{i},
$$

where the surface gradients of the shape functions $\operatorname{Grad} \phi_{i}^{(k)}$ are constant vectors.

Now the unknown values $g_{j}$ can be found from the least-squares procedure:

$$
\Psi=\sum_{i=1}^{N}\left|\gamma_{i}-\gamma_{i}^{*}\right|^{2} \rightarrow \min .
$$

Taking partial derivatives of $\Psi$ with respect to $g_{j}, j=1, \ldots, M$, and making them equal to zero, we obtain linear system of $M \times M$ size with symmetric, but not positively-defined matrix. Moreover, it is clear, that this system is singular (in practice, due to the truncation errors, it can be non-singular, but ill-conditioned), that follows from the fact, that the potential density value can be chosen arbitrary at some specified point. To be more specific, we assume $g_{M}=0$, that means that the last row and last column in the least-squares matrix should be nullified, the diagonal coefficient can be chosen arbitrary (non-zero) and the last coefficient in the right-hand side also should be nullified. The resulting regularized matrix is now symmetric and positively defined.

\section{NUMERICAL RESULTS}

In order to estimate the accuracy of the developed technique of singularity exclusion and its analytical integration, some model problems were considered for the bodies, shown in Fig. 3. For all the bodies (sphere, wing of the finite span, complex-shaped body) unstructured essentially non-uniform triangular mesh was generated by using preprocessor SALOME and NETGEN algorithm. Some parameters of the surface meshes are given in the Table 1. 

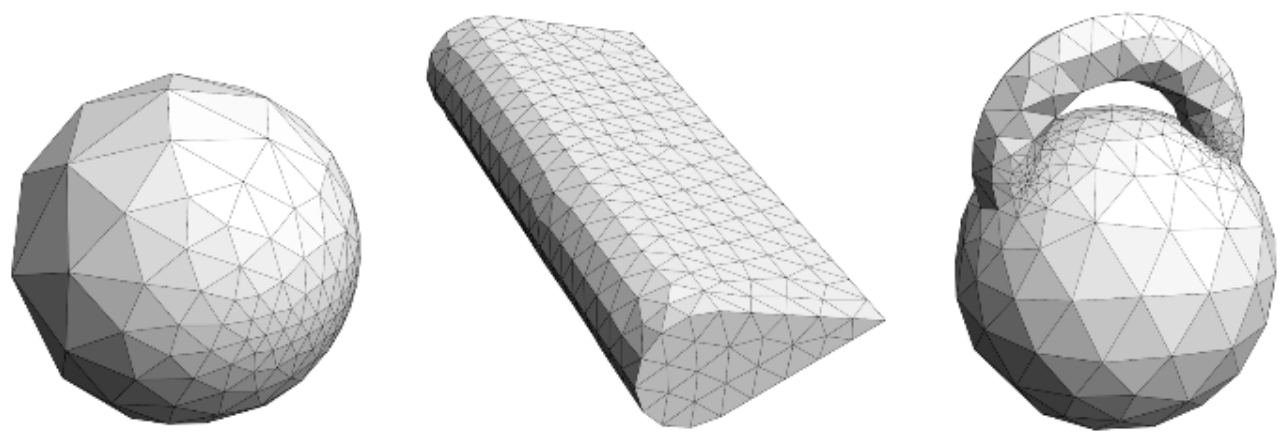

Figure 3: Sphere, wing of the finite span, complex-shaped body and the surface triangular meshes on their surfaces.

Table 1: Mesh parameters and relative errors for the coefficients $\mathbf{I}_{i j}$ calculation.

\begin{tabular}{|l|c|c|c|}
\hline & Sphere & Wing & Complex-shaped body \\
\hline Number of cells & 342 & 742 & 636 \\
\hline Max cells area ratio & 33.9 & 2.9 & 1007.9 \\
\hline $\begin{array}{l}\text { Max cells area ratio for } \\
\text { neighbouring cells }\end{array}$ & 3.4 & 2.9 & 9.3 \\
\hline Max cells sides ratio & 2.0 & 1.7 & 2.4 \\
\hline Min/max cell angle & $30.2^{\circ} / 98.7^{\circ}$ & $35.6^{\circ} / 91.9^{\circ}$ & $22.4^{\circ} / 122.6^{\circ}$ \\
\hline$\delta$, method 1 & 0.40 & 2.28 & 0.65 \\
\hline$\delta$, method 2 & 0.054 & 0.053 & 0.052 \\
\hline$\delta$, method 3 & 0.00092 & 0.0018 & 0.00098 \\
\hline
\end{tabular}

The exact values of the $\mathbf{I}_{i j}$ coefficients are unknown, so instead of exact ones we considered approximate numerical values of the corresponding integrals (13), calculated by using computer algebra system Wolfram Mathematica to be reference values.

In Table 1 the values of the maximal relative error

$$
\delta=\max _{i}\left(\frac{\max _{j}\left|\mathbf{I}_{i j}-\mathbf{I}_{i j}^{*}\right|}{\max _{j}\left|\mathbf{I}_{i j}^{*}\right|}\right), \quad i, j=1, \ldots, N,
$$

are given for three different methods of the integrals (13) calculation:

1. By using formula (13), where for $\mathbf{J}_{j}(\mathbf{r})$ calculation in (11) also the Gaussian quadrature formulae are used;

2. According to formulae (13), where the inner integral $\mathbf{J}_{j}(\mathbf{r})$ is calculated analytically (exactly) using formula (12);

3. Semi-analytically according to the developed technique of singularity exclusion and its analytical integrating for the mesh cells having common edge or common vertex. 
It is seen, that the developed technique allows for significant improvement of the accuracy of the boundary integral equation approximation.

\section{CONCLUSION}

A procedure of the vortex sheet intensity reconstruction is proposed, which consists of two steps. Firstly, the boundary integral equation of the second order is solved by using Galerkin approach, which expresses the equality between the tangential components of flow velocity limit value and the body surface velocity. Secondly, the least-squares procedure is implemented, which permits one to find nodal values of the double layer potential and then reconstruct divergence-free vortex sheet intensity distribution.

For the coeffcients of the linear system, which approximates the boundary integral equation, a semi-analytical approach is suggested, which allows for their calculation with high accuracy. It is shown that the developed algorithm permits one to improve significantly the accuracy of solution for the complex-shaped bodies with low-quality surface meshes.

This procedure be used in vortex methods implementations where it is necessary to simulate vorticity generation process on the body surface at every time step.

\section{ACKNOWLEDGEMENT}

The research was supported by the Russian Science Foundation (grant No. 17-79-20445).

REFERENCES
[1] Cottet, G.-H. \& Koumoutsakos, P., Vortex Methods: Theory and Practice, Cambridge: Cambridge University Press, 2000.

[2] Lifanov, I.K., Poltavskii, L.N. \& Vainikko, G.M., Hypersingular Integral Equations and their Applications, Boca Raton: CRC Press, 2003.

[3] Kempka, S.N., Glass, M.W., Peery, J.S., Strickland, J.H. \& Ingber, M.S., Accuracy consideration for implementing velocity boundary conditions in vorticity formulations. SANDIA REPORT, SAND96-0583 UC-700, 52 p., 1996.

[4] Saffman, P.G., Vortex Dynamics. Cambridge: Cambridge University Press, 1992.

[5] Timofeev, V.N., Mathematical simulation of the subsonic flow around the lengthening bodies with the flow separation in the region of ground shear with the use of an equivalent body. Journal of Physics: Conference Series, 1141, art. 012095, 2018.

[6] Weissmann, S. \& Pinkall, U., Filament-based smoke with vortex shedding and variational reconnection. ACM Trans. Graph. 29(4), pp. 1, 2010.

[7] Shcheglov, G.A. \& Dergachev, S.A. Hydrodynamic loads simulation for 3D bluff bodies by using the vortex loops based modification of the vortex particle method. Proc. of V Intern. Conf. on Particle-based Methods PARTICLES 2017, pp. 725-731, 2017.

[8] Shcheglov, G.A. \& Dergachev, S.A., Vortex loops based method for subsonic aerodynamic loads calculation. 3rd International Conference on Design and Manufacturing Engineering, 221, art. 05004, 2018.

[9] Marchevsky, I.K. \& Shcheglov, G.A., Efficient semi-analytical integration of vortex sheet influence in $3 \mathrm{~d}$ vortex method. Proceedings of the V International Conference on Particle-based Methods PARTICLES 2017, pp. 703-714, 2017.

[10] Marchevsky, I.K. \& Shcheglov, G.A., Semi-analytical influence computation for vortex sheet with piecewise constant intensity distribution in 3D vortex methods. Proceedings of the 6th European Conference on Computational Mechanics (ECCM 6), 7th European Conference on Computational Fluid Dynamics (ECFD 7), 2018. 
www.eccm-ecfd2018.org/admin/files/filePaper/p2059.pdf. Accessed on: 10 Mar. 2019.

[11] Marchevsky, I., Dergachev, S.A. \& Shcheglov, G.A., New modification of 3D meshless Lagrangian vortex method with improved boundary condition satisfaction and divergence-free vorticity representation. Proceedings Topical Problems of Fluid Mechanics 2019, pp. 151-160, 2019.

[12] Kuzmina, K.S., Marchevskii, I.K. \& Moreva, V.S., Vortex sheet intensity computation in incompressible flow simulation around an airfoil by using vortex methods,. Mathematical Models and Computer Simulations, 10(3), pp. 276-287, 2018.

[13] Kuzmina, K.S., Marchevsky, I.K. \& Ryatina, E.P., Exact analytical formulae for linearly distributed vortex and source sheets influence computation in 2D vortex methods. Journal of Physics: Conference Series, 918(1), art. 012013, 2017.

[14] Brebbia, C.A., Telles, J.C.F. \& Wrobel, L.C., (eds), Boundary Element Techniques, Berlin and New York: Springer-Verlag, 1984.

[15] Reid, M.T.H, White, J.K. \& Johnson, S.G., Generalized Taylor-Duffy method for efficient evaluation of galerkin integrals in boundary-element method computations. IEEE Transactions on Antennas and Propagation, 63(1), pp. 195-209, 2015.

[16] Gradshteyn, I.S., Ryzhik, I.M., Geronimus, Y.V., Tseytlin, M.Y. \& Jeffrey, A., Table of Integrals, Series, and Products. Academic Press, 2015.

[17] Van Oosterom, A. \& Strackee, J. The solid angle of a plane triangle. IEEE Transactions on Biomedical Engineering, BME-30, pp. 125-126, 1983.

[18] Zienkiewicz, O.C. \& Taylor, L.R. The Finite Element Method. Volume 1: The Basis. Butterworth-Heinemann, 2000. 